\title{
The Challenges of Flavour Physics
}

\author{
Gino Isidori*i \\ INFN, Laboratori Nazionali di Frascati, Via E. Fermi 40 I-00044 Frascati, Italy. \\ E-mail: Gino.Isidori@lnf.infn.it
}

The open problems and the most recent developments in flavour physics are briefly reviewed. Particular attention is devoted to the current "anomalies" in the CKM picture and their possible interpretation in beyond-the-Standard-Model frameworks.

To the memory of Nicola Cabibbo.

35th International Conference of High Energy Physics - ICHEP2010,

July 22-28, 2010

Paris France

\footnotetext{
* Speaker.

${ }^{\dagger}$ Work supported by the Technische Universität München - Institute for Advanced Study, funded by the German Excellence Initiative.
} 


\section{Introduction: the big challenges in flavour physics}

A lot of progress has been made since 1963, when the first building block of what we now call flavour physics was laid down [1]. The mechanism of flavour mixing has been tested with high accuracy in the quark sector, where all flavour-violating phenomena seems to be well described by the Standard Model (SM) Yukawa interaction with three generations of quarks [2]. Flavour mixing has been observed also in the neutrino sector, indicating the existence of a non-vanishing neutrino mass matrix which cannot be accommodated within the SM.

Despite this important progress, the origin of flavour is still a mystery. Our "ignorance" can be summarized by the following two open questions:

- What determines the observed pattern of masses and mixing angles of quarks and leptons?

- Which are the sources of flavour symmetry breaking accessible at low energies? Is there anything else beside the SM Yukawa couplings and the neutrino mass matrix?

The attempts to answer the first question are typically based on the introduction of a non-trivial flavour dynamics at some high scale (see e.g. [3]). The new dynamics can be associated to Abelian or non-Abelian continuous symmetries (see e.g. [4] for a recent discussion) or, as suggested by the neutrino sector, to a discrete symmetry (see e.g. [5]). Alternatively, in models with extra spacetime dimensions, the flavour hierarchy could be an infrared-property associated to the different localization of the fermion profiles in extra dimensions (see e.g. [6]). In all cases it is quite easy to reproduce the observed mass matrices in terms of a reduced number of free parameters, while it is difficult to avoid problems with Flavour Changing Neutral Currents (FCNCs) unless some amount of fine-tuning is introduced. Most important, it is not easy to make progress in answering this question without knowing the ultraviolet completion of the model.

Answering the second question is more easy: it is mainly a question of precision, both on the theory and on the experimental side. Despite the phenomenological success of the SM, we have clear indications that this theory needs an ultraviolet completion. The most realistic proposals point toward the existence of new degrees of freedom at the $\mathrm{TeV}$ scale, possibly accessible at the high- $p_{T}$ experiments at the LHC. In this perspective the second question above is related to the flavour structure of these new degrees of freedom: is flavour symmetry breaking at the $\mathrm{TeV}$ scale fully controlled by the SM Yukawa couplings and the neutrino mass matrix? As far as the effects in low-energy observables are concerned, this question can be formulated within a general effective theory approach and, to a large extent, it is independent from the ultraviolet dynamics (see e.g. [7]). Following this approach we have already learned a lot about the possible flavour structure of physics beyond the SM. As I will illustrate in the rest of this talk, we could learn even more improving the precision in selected low-energy observables.

\subsection{Present status of CKM fits: the global picture}

The good overall consistency of the experimental constraints appearing in the so-called CKM fits (see $[8,9]$ for updated results) indicate that there is not much room for new sources of flavour symmetry breaking accessible at low energies (see Fig. 1). The success of the SM in describing flavour mixing is also confirmed by a series of other observations. Two notable examples are: 

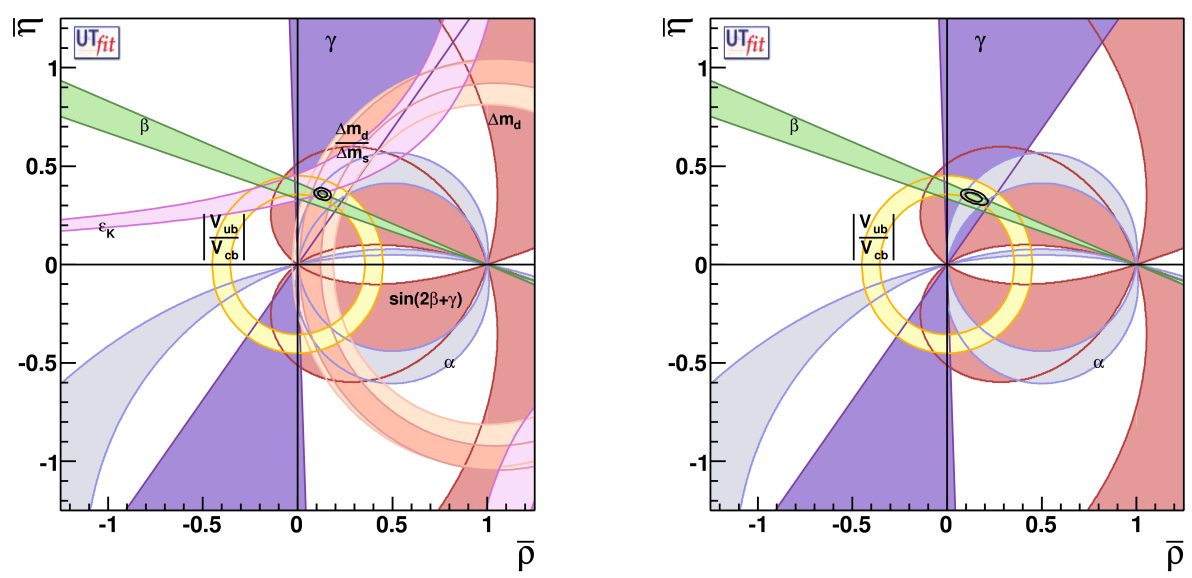

Figure 1: Fits of the CKM unitarity triangle from Ref. [8]. Left: SM case. Right: CKM constraints in extensions of the SM satisfying the MFV hypothesis (universal unitarity triangle [10]).

i) the agreement between the SM prediction and the experimental determination of $\mathscr{B}\left(B \rightarrow X_{s} \gamma\right)$, where both theory and experimental errors are below the $10 \%$ level [11, 12]; ii) the test of the CKM unitarity relation $\left|V_{i d}\right|^{2}+\left|V_{u s}\right|^{2}+\left|V_{Y b}\right|^{2}=1$, which is presently probed below the per-mil level [13]. All these precise tests can be translated into stringent bounds on physics beyond the SM (see [14]). These bounds allow us to conclude that new flavor-breaking sources comparable and not aligned to the SM Yukawa couplings are excluded for new degrees of freedom at the TeV scale.

The absence of large deviations form the SM in flavour-changing processes, together with the need of new degrees of freedom at the TeV scale to stabilize the SM Higgs sector, is the main motivation for the so-called Minimal Flavour Violation (MFV) hypothesis. Under this assumption, the SM Yukawa couplings are the only flavour symmetry breaking terms also beyond the SM [15]. More precisely, in the limit of vanishing quark Yukawa couplings the effective Lagrangian describing both SM and new degrees of freedom is invariant under the global quark flavour symmetry $S U(3)_{Q_{L}} \times S U(3)_{D_{R}} \times S U(3)_{U_{R}}$ [16]. Employing this hypothesis non-standard contributions in flavour-violating transitions turn out to be suppressed to a level consistent with experiments even for New Physics (NP) in the TeV range [15]. The MFV hypothesis provides the technical tool to address the second question listed in the Introduction: if MFV holds, then there are no other sources of flavour symmetry breaking accessible at low energies. Two comments are in order:

- The MFV ansatz is quite successful on the phenomenological side; however, it is unlikely to be an exact property of the model valid to all scales. Despite some recent attempts to provide a dynamical justification of this symmetry-breaking ansatz (see e.g. [17, 18]), the most natural possibility is that MFV is only an accidental low-energy property of the theory [19]. It is then very important to search for possible deviations (even if tiny) from the MFV predictions.

- Even if the MFV ansatz holds, it does not necessarily imply small deviations from the SM predictions in all flavour-changing phenomena. The MFV ansatz can be implemented in different ways. For instance, in models with two Higgs doublets we can change the relative 
normalization of the two Yukawa couplings [15], we can decouple the breaking of CP invariance from the breaking of the $S U(3)_{Q_{L}} \times S U(3)_{D_{R}} \times S U(3)_{U_{R}}$ quark-flavour group [20] and, in models with strong dynamics at the $\mathrm{TeV}$ scale, we can consider operators with a large number of Yukawa insertions [20]. All these variations leads to different and well defined patterns of possible deviations from the SM that we have just started to investigate.

\section{Recent phenomenological challenges to the CKM picture}

As discussed in the previous Section, the overall picture of quark flavour mixing shows a good consistency with the SM predictions. However, looking more closely, there are a few cases where the agreement is not so good. The most interesting "anomalies" that have emerged in the last few years are: i) the $\sin 2 \beta$ tension in the CKM fit; ii) CP violation (CPV) in $B_{s}$ mixing; iii) the $B \rightarrow \tau v$ branching ratio. These (minor) deviations from the CKM picture are particularly interesting since on the one hand the NP contributions are small and compatible with the absence of large NP signals in other observables, on the other hand the theory errors of the SM predictions are small and/or can be systematically improved in the near future.

\subsection{The $\sin 2 \beta$ tension in the CKM fit}

Within the SM the time-dependent CP asymmetry in $B_{d} \rightarrow \psi K$ (denoted $S_{\psi K}$ ) is expected to be equal to $\sin (2 \beta)$ (up to corrections below the $1 \%$ level), where $\beta=\arg \left[-V_{t d}^{*} V_{t b} /\left(V_{c d}^{*} V_{c b}\right)\right]$ (green band in Fig. 1 left). At present the experimental determination of $S_{\psi K}$ is about $2 \sigma$ lower that the indirect determination of $\sin (2 \beta)$ from other observables $[8,9]$. This problem, which has been noted first in [21,22], could be explained by a NP contribution to the $B_{d}$ mixing amplitude. A detailed statistical analysis of this possibility has recently performed in $[8,23]$. Following the notation of [23], the NP contribution to $B_{q}$ mixing amplitudes are parametrized by the complex quantities $\Delta_{q}$, defined by

$$
M_{12}^{q}=\Delta_{q} \times\left(M_{12}^{q}\right)_{\mathrm{SM}}, \quad 2 M_{B_{q}} M_{12}^{q}=\left\langle\bar{B}_{d}\left|\mathscr{H}_{\mathrm{eff}}\right| B_{d}\right\rangle^{*}, \quad q=d, s,
$$

such that the SM limit is recovered for $\Delta_{q}=1$. Flavour observables are then fitted assuming NP contributes significantly only to $B_{d}$ and $B_{s}$ mixing. The best fit value of $\Delta_{d}$ is shown in the left plot in Fig. 2. As can be seen, the SM point is more than $2 \sigma$ off the best fit value (a similar conclusion has been obtained also in [8]).

While this deviation from the SM is not statistically significant yet, the magnitude of the effect is tantalizing for many realistic NP models. In order to improve the significance of this test we would need to decrease the error on the indirect determination of $\sin (2 \beta)$ via better determinations of $\left|V_{u b}\right|, \gamma$, and the " $\varepsilon_{K}$ band". As far as $\varepsilon_{K}$ is concerned, there is a potential warning because of an irreducible theoretical error associated to long-distance effects. However, as recently show in [24], this error do not exceed the $2 \%$ level and it is still largely sub-dominant.

Note that the best fit value of $\Delta_{d}$ indicates a non-vanishing NP phase in $B_{d}$ mixing. If statistically significant, this would unambiguously indicates the presence of new CPV phases in addition to the unique non-trivial CPV phase in the CKM matrix. 

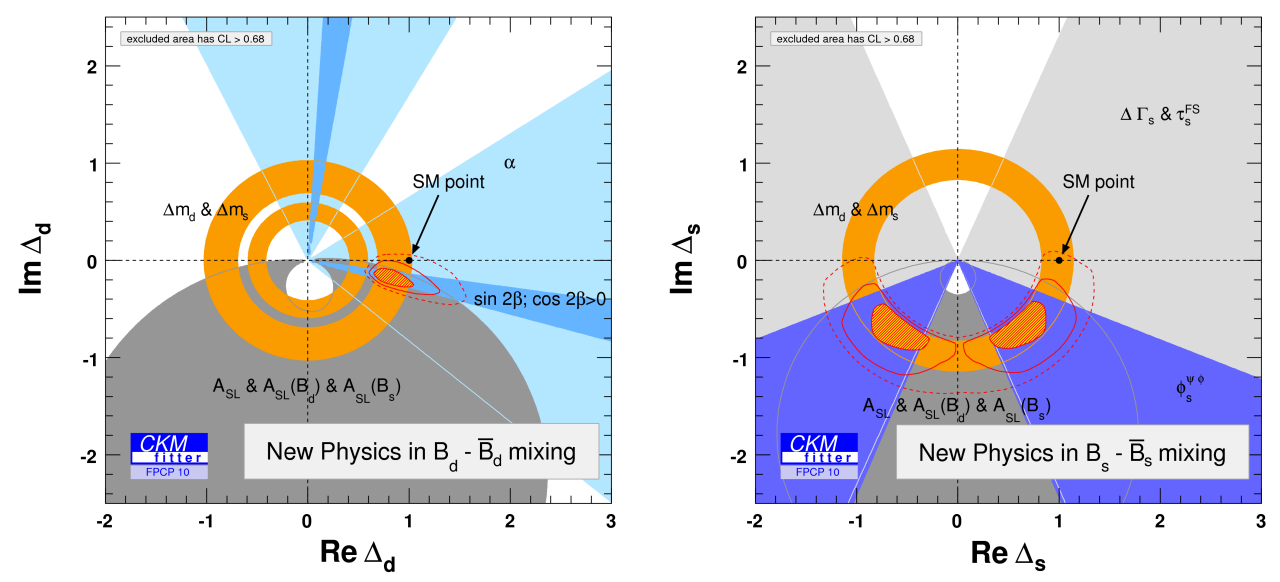

Figure 2: Constraints on NP in the $\left(\operatorname{Re} \Delta_{q}, \operatorname{Im} \Delta_{q}\right)$ planes [23]. In both plots the three red curves (filled, plain, dashed) correspond to 1,2 and $3 \sigma$ contours. The right plot (NP in $B_{s}$ mixing) includes the measurement of $a_{\mathrm{SL}}^{b}$ by D0 but not the very recent $\mathrm{D} 0\left(6.1 f b^{-1}\right)$ and $\operatorname{CDF}\left(5.2 f b^{-1}\right)$ data on $S_{\psi \phi}$.

\subsection{CPV in $B_{s}$ mixing}

Within the SM the $B_{s}$ mixing amplitude $\left(M_{12}^{S}\right)$ has a vanishing CPV phase relative to all the leading (tree-level) $B_{s}$ decay amplitudes. The relative phase between $M_{12}^{s}$ and the nonleptonic decay amplitude $b \rightarrow c \bar{c} s$ can be determined experimentally via the $B_{d} \rightarrow \psi K$ time-dependent CP asymmetry $\left(S_{\psi \phi}\right)$. Moreover, the relative phase between $M_{12}^{s}$ and $\Gamma_{12}^{s}$ can be determined by the semileptonic asymmetry $a_{S L}^{S}$ (see $[23,25,26]$ for more details). The null result expected in both cases is a very clean test of the SM.

Since 2008 both CDF and D0 have started to provide a series of measurements $S_{\psi \phi}$, while only very recently D0 has provided the first determination of $a_{S L}^{s}$. More precisely, D0 has measured a linear combination of $a_{S L}^{s}$ and $a_{S L}^{d}\left(a_{S L}^{b} \approx 0.5 a_{S L}^{s}+0.5 a_{S L}^{d}\right)$ that, combined with $B$-factories data on $a_{S L}^{d}$, allow us to determine $a_{S L}^{s}$ [27]. The central values of the various measurements tend to favor a non-vanishing $B_{s}$ mixing phase, such that $S_{\psi \phi}$ is positive and of order 1. The situation before the results presented at this conference is summarized by the right plot in Fig. 2, where the SM is about $3 \sigma$ off. After the inclusion of the high-statistics data on $S_{\psi \phi}$ by both CDF and D0 announced at this conference [26], the deviation is expected to be slightly less than $2 \sigma$ (an official combination is not yet available).

In this case the error is purely of experimental nature, and substantial improvement is expected in the near future, not only by the Tevatron but also by LHCb. Similarly to the case of $\Delta_{d}$, also the best fit value of $\Delta_{s}$ indicates a non-vanishing NP phase. However, in the $B_{s}$ case the relative deviation from the SM is substantially larger. If confirmed, this non-universal pattern of deviations from the SM in $B_{s}$ and $B_{d}$ mixing would provide a very powerful tool to discriminate possible NP models (see Sect. 3). 


\subsection{The $B \rightarrow \tau \nu$ decay}

The purely leptonic $B \rightarrow \ell v$ decays are particularly interesting for two main reasons. On the one hand they are theoretically very clean: all hadronic uncertainties are confined to the $B$ meson decay constant $\left(f_{B}\right)$, which can be computed reliably using Lattice QCD. On the other hand, the strong helicity suppression makes them particularly sensitive probes of physics beyond the SM, especially of a non-standard Higgs sector.

The $\tau$ channel is the only decay mode of this type observed so far. The expectation for the branching ratio within the SM has the following simple expression,

$$
\mathscr{B}(B \rightarrow \tau v)^{\mathrm{SM}}=\frac{G_{F}^{2} m_{B} m_{\tau}^{2}}{8 \pi}\left(1-\frac{m_{\tau}^{2}}{m_{B}^{2}}\right)^{2} f_{B}^{2}\left|V_{u b}\right|^{2} \tau_{B} .
$$

Using the best fit value of $\left|V_{u b}\right|$ from global CKM fits, and combining both direct lattice QCD constraints and indirect constrains from global CKM fits on $f_{B}$ [28], the UTfit collaboration obtains [8] $\mathscr{B}(B \rightarrow \tau v)^{\mathrm{SM}}=(0.79 \pm 0.07) \times 10^{-4}$. This is substantially lower with respect to the current experimental world average, $\mathscr{B}(B \rightarrow \tau v)^{\exp }=(1.68 \pm 0.31) \times 10^{-4}$, with a statistical significance of a deviation from the SM close to $3 \sigma$. Even taking into account the more conservative estimate of the SM error quoted in [29], the deviation exceed the $2 \sigma$ level.

Beside possible experimental improvements on $B \rightarrow \tau \nu$, an important ingredient to improve the significance of this SM test is the determination of $\left|V_{u b}\right|$ (that is relevant also for the $\sin 2 \beta$ problem discussed before). Both these goals are possible at super- $B$ factories. As far as $\left|V_{u b}\right|$ is concerned, this could be systematically improved in the future with more precise experimental data on $B \rightarrow \pi \ell v$ combined with Lattice QCD results on the $B \rightarrow \pi$ form-factor. Similarly to the approach presently adopted for the determination of $\left|V_{u s}\right|$, the most promising strategy is the experimental determination of the kinematical dependence of the $B \rightarrow \pi$ form-factor combined with Lattice data to fix its overall normalization (see e.g. [30]).

\section{Possible beyond-the-SM explanations of these "anomalies"}

None of the deviations from the SM discussed above has a high statistical significance; however, it is tantalizing to interpret them as possible hints of physics beyond the SM. Several attempts in this direction have been made in the recent literature. In the following I will focus on three classes of models where there has been considerable activity in the last few months, and which are quite interesting because of clear correlations among various observables: i) the two Higgs doublet model (2HDM) with MFV and flavour-blind CPV phases; ii) an effective theory with right-handed (RH) currents; ii) the SM model with four generations of quarks. None of these set-up represents a complete ultraviolet (UV) completion of the SM; however, all of them can be viewed as "simple" effective theories which could arise as the low-energy limit of more ambitious and more complete theories.

\subsection{The $2 \mathrm{HDM}_{\overline{\mathrm{MFV}}}$ framework}

Two or more Higgs doublets are naturally expected in several UV completions of the SM, such as its minimal supersymmetric extension (MSSM). On general grounds, multi-Higgs models 

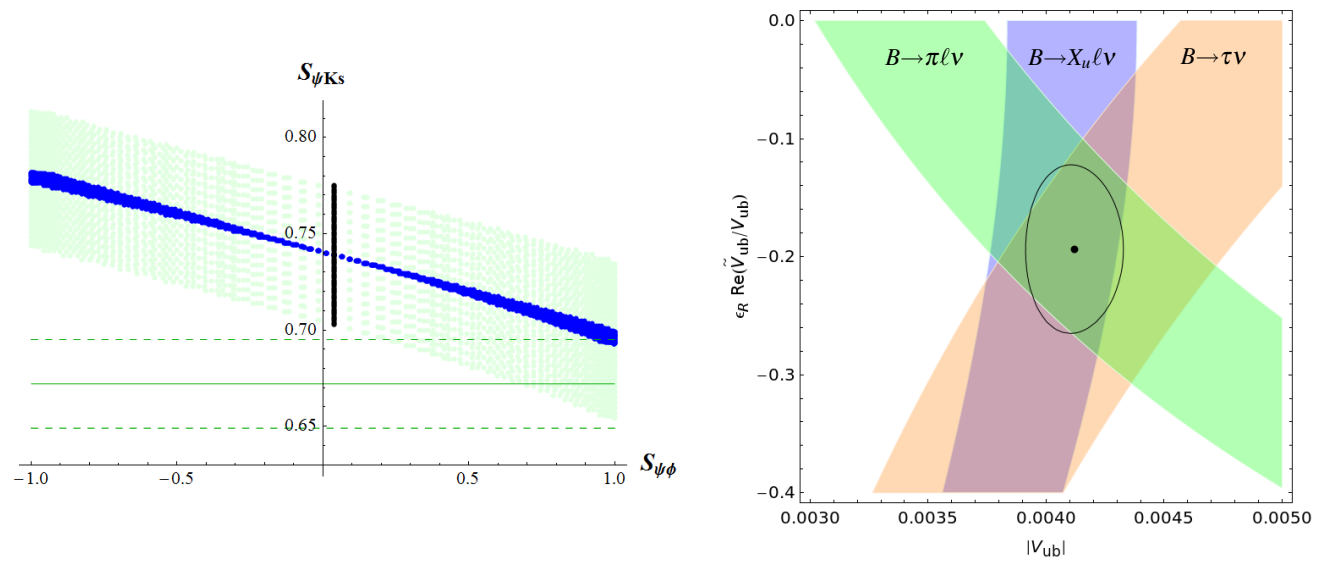

Figure 3: Left: correlation between $S_{\psi K_{S}}$ and $S_{\psi \phi}$ in the $2 \mathrm{HDM}_{\overline{\mathrm{MFV}}}$ [34] (the horizontal lines denote the experimental data, the black vertical line is the SM prediction). Right: constraints on $\left|V_{u b}\right|$ and the relative contribution from RH currents from $B \rightarrow \pi \ell v$ (green), $B \rightarrow X_{u} \ell v$ (blue), and $B \rightarrow \tau \nu$ (orange) [38].

suffer of too-large FCNCs, unless some extra protection mechanism is invoked (see e.g. [31, 32, $33,34]$ for a recent discussion). As already mentioned, an elegant way to justify the smallness of deviations from the SM in flavour-changing observables is provided by the MFV hypothesis. The $2 \mathrm{HDM}_{\overline{\mathrm{MFV}}}$ framework, as defined in [34], is nothing but the most general $2 \mathrm{HDM}$ (with two Higgses of hypercharge $\pm 1 / 2$ ) compatible with the MFV principle (flavour symmetry broken only by two Yukawa couplings), with possible new flavour-blind CPV phases.

This set-up is quite effective in suppressing FCNCs to a level consistent with experiments in most cases, leaving open the possibility of sizable non-standard effects only in specific observables sensitive to Higgs-mediated FCNCs. The effective coupling controlling down-type Higgs-mediated FCNCs $\left(d_{R}^{i} \rightarrow d_{L}^{j} H\right)$ is suppressed both by the CKM combination $V_{t i}^{*} V_{t j}$ (similarly to FCNC amplitudes in the SM) and by the Yukawa coupling of the right-handed quark involved. This double suppression mechanism implies a well-defined pattern of deviations from the SM in $\Delta F=2$ amplitudes such that the largest corrections are expected in $B_{S}$ mixing [20]. Moreover, once the free parameters of the model are tuned to accommodate a large and positive $S_{\psi \phi}$, a small negative correction to $S_{\psi K}$ is automatically implied (see Fig. 3 left), with the ratio of the CPV phases in $\Delta_{s}$ and $\Delta_{d}$ unambiguously linked to $m_{d} / m_{s}$ [34]. This predictive pattern of deviations from the SM is quite interesting in view of the present data on $B_{s, d}$ mixing illustrated in the previous Section (see Fig. 2).

Within an effective theory approach, the region of parameters necessary to fit a large CPV phase in $B_{s}$ mixing is quite natural (heavy Higgs masses of the order of a few $100 \mathrm{GeV}$ ). The new CPV phases are also compatible with present bounds from electric dipole moments (edms) [35]. However, in order to generate a large CPV phase in $B_{s}$ mixing a specific pattern of interference of effective operators with several Yukawa insertions is needed [34, 25]. This is not possible in the standard MSSM, but it could be realized in different underlying models, such as the up-lifted MSSM [36]. The "smoking-gun" of this framework are $\mathscr{B}\left(B_{s, d} \rightarrow \ell^{+} \ell^{-}\right)$largely enhanced over their SM expectations, with $\mathscr{B}\left(B_{s} \rightarrow \ell^{+} \ell^{-}\right) / \mathscr{B}\left(B_{d} \rightarrow \ell^{+} \ell^{-}\right)$fixed to its SM value, and edms close to their present bounds [35]. 


\subsection{Right-handed currents}

One of the main properties of SM is the left-handed nature of the flavour-violating chargedcurrent interactions. This property could well be modified in extensions of the SM, such as leftright symmetric models, or even Higgs-less models. A recent phenomenological interest in flavourviolating RH charged currents originates from the tensions between inclusive and exclusive determinations of $\left|V_{u b}\right|$. As pointed out in particular in [37], the presence of RH currents could either remove or significantly weaken these tensions.

Beside a clear benefit in $b \rightarrow u \ell v$ transitions (see the right panel in Fig. 3), it is interesting to understand if RH currents are compatible with constraints from other processes and where else they could show up. This problem can been addressed in general terms by means of an effective theory approach. In particular, in Ref. [38] it has been considered an effective theory based on the underlying $S U(2)_{L} \times S U(2)_{R} \times U(1)_{B-L}$ global electroweak symmetry, with the same low-energy particle content as in the SM, and with a left-right symmetric flavour group broken only by two Yukawa couplings (RHMFV framework). A central role in this theory is played by a new unitary matrix $(\tilde{V})$ that controls flavour-mixing in the RH sector. This matrix, and the overall strength of RH currents $\left(\varepsilon_{R}\right)$, can be constrained by all available data on semileptonic decays. Interestingly, a sizable contribution to $b \rightarrow u$ (Fig. 3 right) is not in contradiction with the tight constraints on $s \rightarrow u$ and $d \rightarrow u$, and the effective scale of RH interactions turns out to be around $3 \mathrm{TeV}$.

As far as FCNCs are concerned, the most interesting implications of the RHMFV framework can be listed as follows: it is possible to generate a large CPV phase in $B_{s}$-mixing (as suggested by data); however, if this condition is fulfilled, no sizable effects in $B_{d}$-mixing are expected. Moreover, RH currents imply a $\mathscr{O}\left(10^{-3}\right)$ deviation in the determination of $\left|V_{u s}\right|$ from $K \rightarrow \pi \ell v$ and $K \rightarrow \ell v$ decays, that is close to the present experimental sensitivity. Finally, non-standard contributions to $B \rightarrow\left\{X_{s}, K, K^{*}\right\} \nu \bar{v}$ and $K \rightarrow \pi \nu \bar{v}$ decays can be significant and, if present, the deviations from the SM in these decays would exhibit a well-defined pattern of correlations [38].

\subsection{Fourth generation}

The addition of a fourth generation of quarks and leptons to the SM is one of the simplest extensions of the SM one can conceive (SM4 framework). The SM4 is not particularly interesting by itself; however, on the one hand it is allowed by electroweak data, and actually it improves the quality of the electroweak fit [39], on the other hand it provides a simple tool to analyse the impact of the mixing between SM and heavy fermions which is expected in several more complete extensions of the SM.

In the last few years a renewed interest in the SM4 has been triggered by the possible impact in flavour-physics observables (see Ref. [40, 41, 42, 43] and references therein). In the quark sector the model contains seven new parameters: two heavy quark masses, three new mixing angles and two new CPV phases. The quark masses are bounded to be below $\sim 600 \mathrm{GeV}$ by the perturbativity of the corresponding Yukawa couplings, and the mass splitting is tightly constrained by electroweak data. Global fits of the new flavour mixing parameters have been performed in [42, 43]. The main results can be summarized as follows: i) the tensions of the SM in $B_{s}$ and $B_{d}$ mixing can both be solved; ii) a large CPV phase in $B_{S}$ mixing necessarily implies a suppression of the time-dependent $\mathrm{CPV}$ asymmetries in $b \rightarrow s$ penguin-type modes, $B \rightarrow \phi K$ and $B \rightarrow \eta^{\prime} K$, in agreement with data (as 

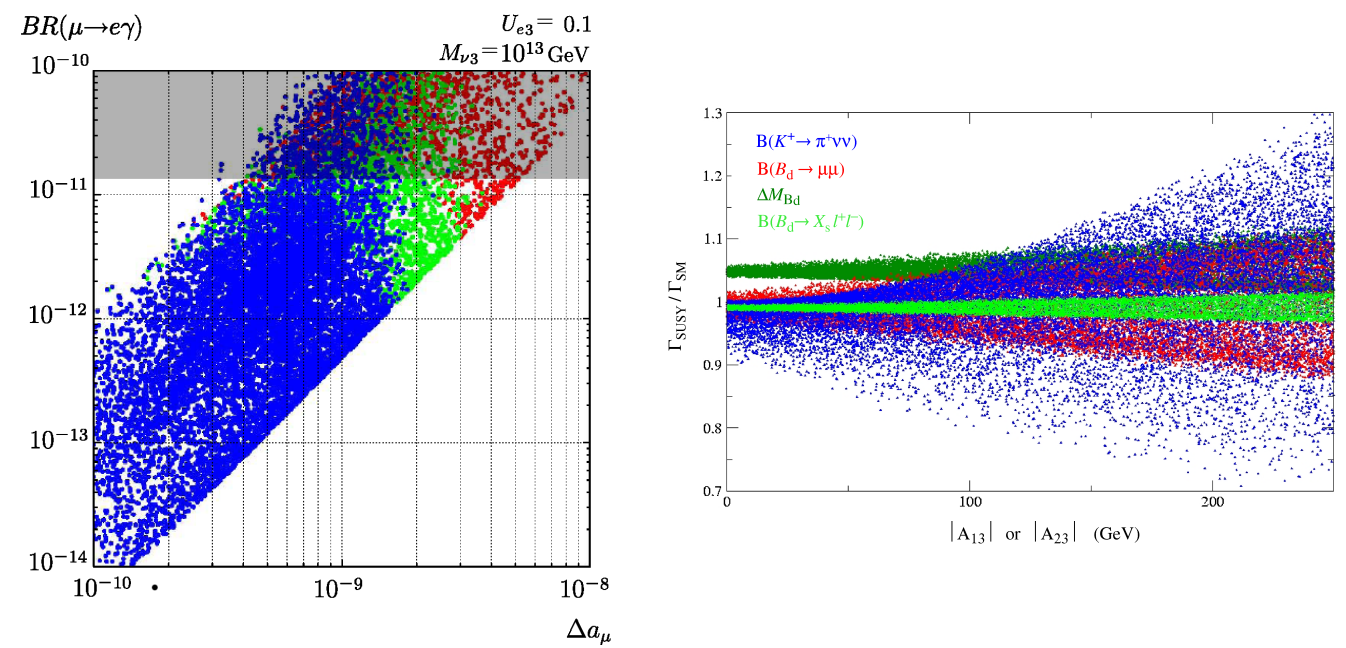

Figure 4: Left: correlation between $\mathscr{B}(\mu \rightarrow e \gamma)$ and $\Delta a_{\mu}$ in the MSSM with heavy RH neutrinos [47]. Right: dependence of various FCNC observables (normalized to their SM value) on the up-type trilinear terms $A_{13}$ and $A_{23}$ (generically denoted by $A_{13}$ ) in the MSSM with non-minimal $A$ terms [52].

noted first in [40]), it also implies a sizable enhancement of $\mathscr{B}\left(B_{s} \rightarrow \mu^{+} \mu^{-}\right)$, with $\mathscr{B}\left(B_{d} \rightarrow \mu^{+} \mu^{-}\right)$ close to its SM value, a clear non-standard pattern that can be tested in the near future; iii) a large CPV phase in $B_{s}$ mixing also implies a tension between the prediction of $\varepsilon^{\prime} / \varepsilon$ and its measurement, although this effect is hidden by the current uncertainty on $K \rightarrow \pi \pi$ hadronic matrix elements; iv) the rare $K \rightarrow \pi v \bar{v}$ decays can be largely enhanced over their SM predictions.

\section{Experimental challenges for the near future}

Current "anomalies" are certainly interesting, but we cannot exclude they will all disappear with higher statistics. Indeed they are not the most natural expectations in the most "conservative" beyond-SM scenarios, such as models with MFV and no extra CPV phases. On the other hand, there are a few other channels where we can expect sizable deviations from the SM even in "conservative" beyond-SM scenarios, and for which we can expect precise experimental results in the near future. Among the most promising channels for the near future it is worth to mention the following three cases: i) the search for lepton flavour violation (LFV) in charged leptons; ii) the very rare FCNC decays $K \rightarrow \pi \nu \bar{v}$; iii) the helicity- suppressed FCNC $B$ decays $B \rightarrow \ell^{+} \ell^{-}$.

The search for LFV in charged leptons is probably the most interesting goal of flavour physics in the next few years. The observation of neutrino oscillations has clearly demonstrated that lepton flavour is not conserved; however, the smallness of neutrino masses provides a strong indication that neutrinos are generated by an underlying dynamics that violates also the total lepton number. The question is if LFV effects can be visible also in other sectors of the theory, or if we can observe LFV in processes which conserve the total lepton number. The most promising low-energy channel is $\mu \rightarrow e \gamma$, currently under investigation at MEG [44]. On general grounds, if the breaking of the total lepton number occurs at a very high energy scale $\left(\Lambda_{\mathrm{LN}}>10^{12} \mathrm{GeV}\right)$, as expected by the smallness of neutrino masses, and the theory has new degrees of freedom carrying lepton-flavour 

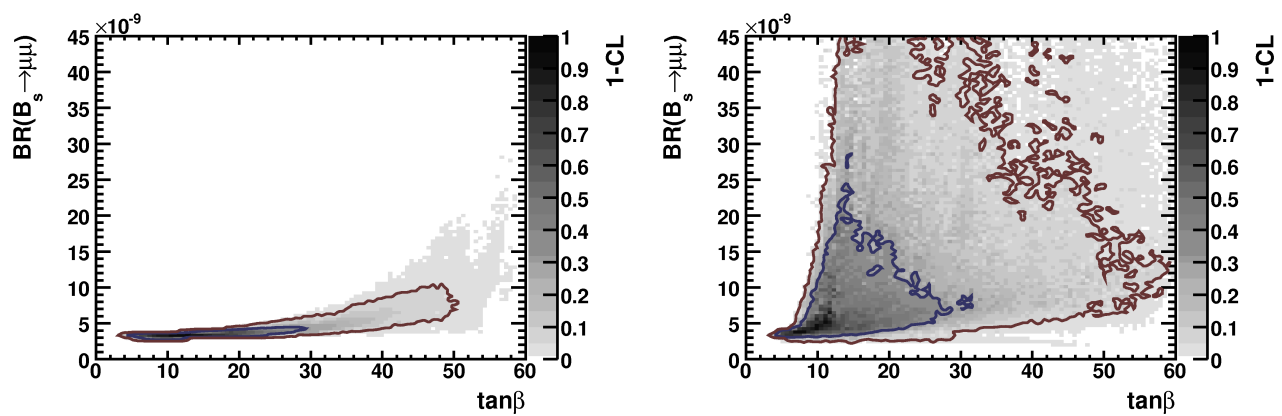

Figure 5: The correlation between $\mathscr{B}\left(B_{s} \rightarrow \mu^{+} \mu^{-}\right)$and $\tan \beta$ in the constrained MSSM (left panel) and in the MSSM with non-universal Higgs soft-mass terms (right panel) [53]. In both panels the CL is obtained combining all the available (indirect) constraints on the model [53].

quantum numbers around the $\mathrm{TeV}$ scale $\left(\Lambda_{\mathrm{LFV}}<10^{4} \mathrm{GeV}\right)$, then $\mu \rightarrow e \gamma$ should be visible. Indeed, employing an effective theory approach with a minimal breaking of lepton flavour, we find [45]

$$
\mathscr{B}(\mu \rightarrow e \gamma) \approx 10^{-13}\left(\frac{\Lambda_{\mathrm{LN}}}{10^{13} \mathrm{GeV}}\right)^{4}\left(\frac{10^{4} \mathrm{GeV}}{\Lambda_{\mathrm{LFV}}}\right)^{4} .
$$

A typical concrete example where this occurs is the MSSM with heavy right-handed neutrinos, where renormalization-group effects generate LFV entries in the left-handed slepton mass matrices at the $\mathrm{TeV}$ scale [46]. Once non-vanishing LFV entries in the slepton mass matrices are generated, LFV rare decays are naturally induced by one-loop diagrams with the exchange of gauginos and sleptons. The flavour-conserving component of the same diagrams induces a non-vanishing contribution to the anomalous magnetic moment of the muon, $\Delta a_{\mu}=\left(g_{\mu}-g_{\mu}^{\mathrm{SM}}\right) / 2$. As shown in Fig. 4 (left), a strong link between these two observable naturally emerges (see e.g. [47]). In this context, the value $\Delta a_{\mu}=\mathscr{O}\left(10^{-9}\right)$, presently indicated by detailed analyses of $g_{\mu}$ [48], reinforce the expectation of $\mu \rightarrow e \gamma$ within the reach of the MEG experiment.

Among the many rare $K, D$, and $B$ decays, the $K \rightarrow \pi \nu \bar{v}$ modes are unique since their SM branching ratios can be computed to an exceptionally high degree of precision, not matched by any other FCNC processes involving quarks (see [49] for the most updated SM predictions). It is then not surprising that these processes continue to raise a strong theoretical interest, both within and beyond the SM (see e.g. [38, 50, 51]). Because of the strong suppression of the $s \rightarrow d$ short-distance amplitude in the SM $\left[V_{t d} V_{t s}^{*}=\mathscr{O}\left(10^{-4}\right)\right]$, rare $K$ decays are the most sensitive probes of possible deviations from the strict MFV ansatz. An illustration of this statement is given in Fig. 4 (right), where the expectations of $\mathscr{B}\left(K^{+} \rightarrow \pi^{+} v \bar{v}\right)$ in the MSSM with non-minimal $A$ terms is shown in comparison with possible deviations from the SM in other rare-decay observables. As can be seen, $\mathscr{B}\left(K^{+} \rightarrow \pi^{+} v \bar{v}\right)$ is the most sensitive probe of this class of models.

The rare decays $B \rightarrow \ell^{+} \ell^{-}$are particularly interesting because in the SM suffer both a strong GIM suppression (being FCNC transitions) and a strong helicity suppression (being two-body leptonic decays). This doubly-suppressed structure make them ideal probes of possible Higgsmediated FCNC amplitudes in models with more than one Higgs doublet, such as the MSSM. Even in the most restricted versions of the MSSM (with MFV and with no new CPV phases), 
$B \rightarrow \ell^{+} \ell^{-}$decays could be largely enhanced over the SM expectations if $\tan \beta$ (the ratio of the two Higgs vevs) is large. The leading non-SM amplitude contributing to $B \rightarrow \ell^{+} \ell^{-}$is generated by the heavy neutral Higgs exchange ( $B \rightarrow A, H \rightarrow \ell^{+} \ell^{-}$). However, since the effective FCNC coupling of the neutral Higgs bosons is generated at the quantum level, the amplitude has a strong dependence on other MSSM parameters in addition to $M_{A, H}$ and $\tan \beta$. In particular, a key role is played by $\mu$ and the up-type trilinear soft-breaking term $\left(A_{U}\right)$, which control the strength of this effective vertex. The leading parametric dependence of the scalar FCNC amplitude from these parameters is

$$
\mathscr{A}_{\text {Higgs }}\left(B \rightarrow \ell^{+} \ell^{-}\right) \propto \frac{m_{b} m_{\ell}}{M_{A}^{2}} \frac{\mu A_{U}}{M_{\tilde{q}}^{2}} \tan ^{3} \beta \times f_{\text {loop }}
$$

For $\tan \beta \gtrsim 30$ and $M_{A} \lesssim 0.5 \mathrm{TeV}$ the neutral-Higgs contribution can easily lead to $\mathscr{O}(10)$ enhancements of $\mathscr{B}\left(B_{s, d} \rightarrow \ell^{+} \ell^{-}\right)$over the SM expectations. Most important, these decays represent a very useful tool to determine a combination of MSSM parameters that would help in discriminating different versions of the model (see e.g. Fig. 5). At present the most significant constraints are obtained from $\mathscr{B}\left(B_{s} \rightarrow \mu^{+} \mu^{-}\right)$, where the experimental upper limit, $\mathscr{B}\left(B_{s} \rightarrow \mu^{+} \mu^{-}\right)<5.8 \times 10^{-8}$ [54], is less than 20 times the SM expectation, $\mathscr{B}\left(B_{s} \rightarrow \mu^{+} \mu^{-}\right)_{\mathrm{SM}}=(3.2 \pm 0.2) \times 10^{-9}$.

\section{Conclusions}

The origin of flavour remains, to a large extent, an open problem. However, a significant progress has been achieved in the phenomenological investigation of the sources of flavour symmetry breaking accessible at low energies. This investigation has allowed to set very stringent constraints on various extensions of the SM, ruling out models with significant misalignments from the SM Yukawa couplings at the TeV scale.

What we learned so far does not imply we cannot see some deviation from the SM in lowenergy process in the near future. A few interesting anomalies in the CKM picture have started to emerge. Some of them will go away with more data, but others may well be the first signals of new physics at the $\mathrm{TeV}$ scale. Even more interesting are the prospects of finding deviations from the SM, or to set very powerful constraints on physics beyond the SM, in clean processes such as $\mu \rightarrow e \gamma, B \rightarrow \ell^{+} \ell^{-}$, and $K \rightarrow \pi v \bar{v}$, where a significant experimental progress is expected in the next few years. As briefly outlined in this talk (see Ref. [55] for a more extensive discussion), once some clear deviation from the SM will be established, the key tool to make progress in this field is to identify correlations among different non-standard effects that can reveal the flavour-breaking pattern of the new degrees of freedom.

\section{Acknowledgment}

This talk is dedicated to the "father" of flavour physics, Nicola Cabibbo, who sadly passed away a few weeks after the end of this conference.

\section{References}

[1] N. Cabibbo, Phys. Rev. Lett. 10, 531 (1963). 
[2] M. Kobayashi and T. Maskawa, Prog. Theor. Phys. 49, 652 (1973).

[3] C. D. Froggatt and H. B. Nielsen, Nucl. Phys. B 147 (1979) 277.

[4] Z. Lalak, S. Pokorski and G. G. Ross, JHEP 1008 (2010) 129 [arXiv:1006.2375 [hep-ph]].

[5] G. Altarelli and F. Feruglio, arXiv:1002.0211 [hep-ph].

[6] Y. Grossman and M. Neubert, Phys. Lett. B 474 (2000) 361 [arXiv:hep-ph/9912408]; K. Agashe, G. Perez and A. Soni, Phys. Rev. D 71 (2005) 016002 [arXiv:hep-ph/0408134].

[7] G. Isidori, PoS E FT09 (2009) 034 [arXiv:0908.0404 [hep-ph]].

[8] A. Bevan et al. [UTfit Collaboration], arXiv:1010.5089 [hep-ph], updated results at: http://www.utfit.org/UTfit/ (talk presented by C. Tarantino at this conference).

[9] J. Charles et al. [CKMfitter Collaboration], Eur. Phys. J. C41, 1-131 (2005) [hep-ph/0406184], updated results at: http://ckmfitter.in2p3.fr (talk presented by S. T'Jampens, at this conference).

[10] A. J. Buras et al. Phys. Lett. B 500 (2001) 161 [arXiv:hep-ph/0007085].

[11] M. Misiak, arXiv:1010.4896 [hep-ph].

[12] T. Hurth and M. Nakao, Ann. Rev. Nucl. Part. Sci. 60 (2010) 645 [arXiv:1005.1224 [hep-ph]].

[13] M. Antonelli et al., Eur. Phys. J. C 69, 399 (2010) [arXiv:1005.2323 [hep-ph]].

[14] G. Isidori, Y. Nir and G. Perez, arXiv:1002.0900 [hep-ph].

[15] G. D’Ambrosio, G. F. Giudice, G. Isidori and A. Strumia, Nucl. Phys. B 645, 155 (2002) [hep-ph/0207036].

[16] R. S. Chivukula and H. Georgi, Phys. Lett. B 188 (1987) 99.

[17] R. Zwicky and T. Fischbacher, Phys. Rev. D 80 (2009) 076009 [arXiv:0908.4182 [hep-ph]].

[18] M. E. Albrecht, T. Feldmann and T. Mannel, JHEP 1010 (2010) 089 [arXiv:1002.4798 [hep-ph]].

[19] B. Grinstein, M. Redi and G. Villadoro, arXiv:1009.2049 [hep-ph].

[20] A. L. Kagan, G. Perez, T. Volansky and J. Zupan, Phys. Rev. D 80 (2009) 076002 [arXiv:0903.1794 [hep-ph]].

[21] E. Lunghi and A. Soni, Phys. Lett. B 666 (2008) 162 [arXiv:0803.4340 [hep-ph]].

[22] A. J. Buras and D. Guadagnoli, Phys. Rev. D 78, 033005 (2008) [arXiv:0805.3887 [hep-ph]].

[23] A. Lenz et al., arXiv:1008.1593 [hep-ph].

[24] A. J. Buras, D. Guadagnoli and G. Isidori, Phys. Lett. B 688 (2010) 309 [arXiv:1002.3612 [hep-ph]].

[25] Z. Ligeti, M. Papucci, G. Perez and J. Zupan, Phys. Rev. Lett. 105 (2010) 131601 [arXiv:1006.0432 [hep-ph]].

[26] G. Borissov, talk presented at this conference.

[27] V. M. Abazov et al. [D0 Collaboration], arXiv:1005.2757.

[28] M. Bona et al. [UTfit Collaboration], Phys. Lett. B 687 (2010) 61 [arXiv:0908.3470 [hep-ph]].

[29] B. Bhattacherjee, A. Dighe, D. Ghosh and S. Raychaudhuri, arXiv:1012.1052 [hep-ph].

[30] M. Antonelli et al., Phys. Rept. 494 (2010) 197 [arXiv:0907.5386 [hep-ph]]. 
[31] G. C. Branco, W. Grimus and L. Lavoura, Phys. Lett. B 380 (1996) 119 [arXiv:hep-ph/9601383]; F. J. Botella, G. C. Branco and M. N. Rebelo, Phys. Lett. B 687 (2010) 194 [arXiv:0911.1753];

[32] A. Pich and P. Tuzon, Phys. Rev. D 80 (2009) 091702 [arXiv:0908.1554 [hep-ph]].

[33] R. S. Gupta and J. D. Wells, Phys. Rev. D 81 (2010) 055012 [arXiv:0912.0267 [hep-ph]].

[34] A. J. Buras, M. V. Carlucci, S. Gori and G. Isidori, JHEP 1010 (2010) 009 [arXiv:1005.5310 [hep-ph]].

[35] A. J. Buras, G. Isidori and P. Paradisi, arXiv:1007.5291 [hep-ph].

[36] B. A. Dobrescu, P. J. Fox and A. Martin, Phys. Rev. Lett. 105 (2010) 041801 [arXiv:1005.4238 [hep-ph]].

[37] A. Crivellin, Phys. Rev. D 81, 031301 (2010) [arXiv:0907.2461 [hep-ph]].

[38] A. J. Buras, K. Gemmler and G. Isidori, Nucl. Phys. B 843 (2011) 107 [arXiv:1007.1993 [hep-ph]].

[39] B. Holdom, W. S. Hou, T. Hurth, M. L. Mangano, S. Sultansoy and G. Unel, PMC Phys. A 3 (2009) 4 [arXiv:0904.4698 [hep-ph]].

[40] G. W.-S. Hou, talk presented at this conference; G. W. S. Hou, M. Nagashima and A. Soddu, Phys. Rev. Lett. 95 (2005) 141601 [arXiv:hep-ph/0503072]; Phys. Rev. D 76 (2007) 016004 [arXiv:hep-ph/0610385].

[41] M. Bobrowski, A. Lenz, J. Riedl and J. Rohrwild, Phys. Rev. D 79 (2009) 113006 [arXiv:0902.4883 [hep-ph]].

[42] A. Soni, A. K. Alok, A. Giri, R. Mohanta and S. Nandi, Phys. Rev. D 82 (2010) 033009 [arXiv:1002.0595 [hep-ph]].

[43] A. J. Buras, B. Duling, T. Feldmann, T. Heidsieck, C. Promberger and S. Recksiegel, JHEP 1009 (2010) 106 [arXiv:1002.2126 [hep-ph]].

[44] A. Baldini, talk presented at this conference.

[45] V. Cirigliano, B. Grinstein, G. Isidori and M. B. Wise, Nucl. Phys. B 728 (2005) 121 [arXiv:hep-ph/0507001].

[46] F. Borzumati and A. Masiero, Phys. Rev. Lett. 57 (1986) 961.

[47] J. Hisano, M. Nagai, P. Paradisi and Y. Shimizu, JHEP 0912 (2009) 030 [arXiv:0904.2080 [hep-ph]].

[48] M. Davier, A. Hoecker, B. Malaescu and Z. Zhang, arXiv:1010.4180 [hep-ph].

[49] J. Brod, M. Gorbahn and E. Stamou, arXiv:1009.0947 [hep-ph].

[50] M. Blanke, A. J. Buras, B. Duling, S. Recksiegel and C. Tarantino, Acta Phys. Polon. B 41 (2010) 657 [arXiv:0906.5454 [hep-ph]].

[51] M. Bauer, S. Casagrande, U. Haisch and M. Neubert, JHEP 1009 (2010) 017 [arXiv:0912.1625 [hep-ph]].

[52] G. Isidori, F. Mescia, P. Paradisi, C. Smith and S. Trine, JHEP 0608 (2006) 064 [arXiv:hep-ph/0604074].

[53] O. Buchmueller et al., Eur. Phys. J. C 64, 391 (2009) [arXiv:0907.5568 [hep-ph]].

[54] T. Aaltonen et al. [CDF Collaboration], Phys. Rev. Lett. 100 (2008) 101802 [arXiv:0712.1708 [hep-ex]].

[55] G. Isidori, arXiv:1001.3431 [hep-ph]; A. J. Buras, arXiv:1012.1447 [hep-ph]. 